\title{
Relations d'Auguste Tissot (1728-1797), médecin à Lausanne, avec le patriciat bernois
}

\author{
Par Marie-Louise Portmann
}

Sous le gouvernement de Berne qui avait conquis le Pays de Vaud en 1536 en faisant la guerre au duc de Savoie, une petite oligarchie indigène s'était formée qui jouissait d'une aisance modeste. A cette classe appartenait le commissaire Pierre Tissot à Grancy qui rendit possible à son fils Auguste l'étude de la médecine à Montpellier, ce qui lui coûta plus de douze mille francs de Suisse ${ }^{1}$. Après être reçu docteur, en 1749, Auguste Tissot, qui avait été à Montpellier l'hôte du professeur François Boissier de Sauvages (1706-1767), commença à pratiquer la médecine à Lausanne. Dans une épidémie de petite vérole, il eut bien du succès, ce qui lui valut l'estime des citoyens et du bailli Albert Tscharner (1700-1771) ${ }^{2}$. Son maître Boissier de Sauvages conseilla à Tissot de s'adresser à Albert de Haller. Boissier écrivit le 15 août 1751: «On assure que Haller va à Berne, profitez de

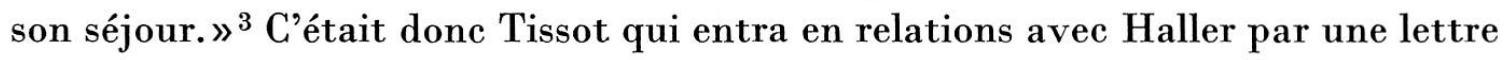
du 22 février 1754 dans laquelle il lui demanda son opinion sur un manuscrit dont le sujet était la variolation ${ }^{4}$. Haller qui était aussi un adhérent de cette nouvelle méthode préventive contre la petite vérole, renvoya le manuscrit un mois plus tard avec ses conseils ${ }^{5}$. Ce fut le début d'une correspondance entre ces deux fameux médecins qui ne se termina qu'à la mort de Haller survenue le 12 décembre 1777. Erich Hintzsche (1900-1975) est l'éditeur de cette vaste correspondance qui nous fait connaître les multiples relations de Tissot avec le patriciat bernois et son engagement au service du gouvernement. Il ressort de cette correspondance que Haller et Tissot avaient besoin l'un de l'autre. Haller savait se servir de Tissot qui traduisit en français des traités du médecin bernois comme par exemple son mémoire sur la sensibilité et l'irritabilité qui avait paru en 1753 en latin ${ }^{6}$. Tissot se donna aussi bien de la peine pour aplanir les difficultés entre le colérique et impatient Haller et ses imprimeurs lausannois Marc-Michel Bousquet, Sigismond d'Arnay et François Grasset. La bonté et la douceur de Tissot facilitèrent toujours ces négociations ${ }^{7}$. En juillet 1763 lorsque Haller fut une fois de plus sur le point de changer d'imprimeur, Tissot lui conseilla de s'adresser à la Société typographique de Berne dont Vincent Bernhard Tscharner (1728-1778) était le fondateur et directeur. Ce furent alors les Tscharner qui prirent soin de l'édition des «Elementa physiologiae» et se soucièrent de les faire imprimer $^{8}$. 
Tissot qui avait à sa disposition la belle bibliothèque de son père et la collection de livres encore plus considérable de son beau-père Jean-François Dapples ${ }^{9}$, professeur à l'Académie de Lausanne, rendit bien des services à Haller en lui prêtant de ses livres et en faisant venir les publications désirées de l'étranger. L'échange fut mutuel, car Haller à son tour fit toujours parvenir à son ami les livres qui l'intéressaient.

Tissot savait aussi se servir de Haller pour faire du bien à ses parents. Lorsqu'en 1759 une cousine de Madame Tissot et le docteur Marc-Béat-Louis-Jacob Porta eurent le désir de se marier bien qu'ils fussent parents, Tissot s'adressa à Haller parce qu'un tel mariage était défendu dans le canton de Berne. Haller parvint à régler l'affaire et le couple fut gracié par le sénat de Berne ${ }^{10}$. En 1765, Tissot recommanda à Haller deux beaux-frères en même temps, ce qui fut fort désapprouvé par Haller ${ }^{11}$. Alors Tissot blâma à son tour la coutume et la nécessité de rechercher par l'intervention de parents ou amis influents les postes vacants au Pays de Vaud ${ }^{12}$. C'était donc le sénat de Berne qui disposait des charges officielles.

Cependant le sujet principal qui préoccupait Tissot c'étaient les problèmes de la médecine et il avait l'intention de remédier aux maux de sa partie. C'est pour cette raison qu'il publia son œuvre principale, l'«Avis au peuple sur sa santé» qui parut en 1761 à Lausanne et qui le rendit célèbre par toute l'Europe. A vrai dire, le travail n'était destiné qu'aux Vaudois, ce qui ressort de la préface à la deuxième édition. Tissot écrit: «Touché du sort des gens du peuple malade dans les campagnes de ce pays, où il périt misérablement par la disette de secours utiles et la multitude de mauvaises directions, mon seul but en écrivant était de prévenir une partie de ces malheurs. Je n'avais destiné ce livre, qui parut pour la première fois au mois de septembre 1761, qu'à une petite enceinte de pays et à un petit nombre de personnes, et je fus très-surpris en apprenant cinq ou six mois après sa publication, qu'il était l'un des livres de science qui eût trouvé le plus de lecteurs. » ${ }^{13} \mathrm{~L}^{\prime}$ "Avis au peuple» valut à Tissot la bourgeoisie de Lausanne et il fut admis dans la Société économique de Berne dont Jean-Rodolphe Tschiffeli (1716-1780) était le fondateur et le président. En outre il reçut du conseil de santé une médaille d'or avec une lettre qui apprécia l'utilité générale de cet ouvrage. Le gouvernement de Berne qui était d'ailleurs peu prodigue de marques d'honneur et de satisfaction, témoigna ainsi à Tissot qu'il avait bien mérité du salut public ${ }^{14}$.

Médecin vaudois, Tissot était responsable vis-à-vis du conseil de santé de Berne qui avait été créé le 18 octobre 1709. C'était un département ou un service de l'hygiène publique qui fut d'abord établi contre pestes et épizooties ${ }^{15}$. Le personnage le plus important dans ce conseil était Albert de Haller. Dans plusieurs 
épidémies, pleurésies bilieuses et fièvres catarrhales à Lausanne et à Aigle, Tissot adressa des rapports au conseil de santé qui souvent demanda ce service au médecin vaudois. En 1765, Tissot fut envoyé à Aigle par le gouvernement pour y donner des directives dans le traitement de l'épidémie de pleurésie bilieuse qui y faisait de grands ravages et le conseil de santé fut bien satisfait des services que Tissot lui rendit. L'attitude modeste du médecin vaudois envers le patriciat bernois est démontrée par la lettre du 25 février 1765 adressée à Haller: «Monsieur de Bonstetten m'écrit qu'il me demandera mon compte pour le voyage d'Aigle, où tout est heureusement fini, ce sera beaucoup m'embarrasser: je n'en ai jamais donné à personne, et il ne sera pas honnête de commencer par le souverain... ${ }^{16}$

A vrai dire, Tissot n'avait pas besoin d'une rémunération de la part du gouvernement puisque ses services médicaux commençaient à être recherchés par des personnages aisés et nobles, par des princes et des princesses qui vinrent de l'étranger à Lausanne pour s'y faire inoculer et pour consulter le fameux médecin. En ce qui concerne ses finances, Tissot fut plus à son aise que le pauvre Haller qui fut souvent dans l'embarras ${ }^{17}$. Aussi Tissot fut en 1770 en état de pouvoir acheter avec son frère la terre Monrion à 45000 frs $^{18}$ alors que Haller s'était vu forcé à renoncer à l'achat en 1763 quand cette terre lui avait été offerte bien meilleur marché ${ }^{19}$.

Outre sa clientèle étrangère Tissot fut aussi consulté par les patriciens bernois. Je ne fais mention ici que de quelques exemples. Quand la femme du bailli de Watteville à Nyon souffrit d'une affection nerveuse et d'obstipation en décembre 1763, Tissot fut son médecin ${ }^{20}$. En 1771, Guillaume Bernard de Muralt (17371796), alors capitaine au régiment d'Erlach, fut phtisique et fut traité par Haller. De Muralt eut le désir de se rendre à Lausanne et il chercha dans le voisinage de Tissot un logement dans un endroit chaud. Le médecin vaudois eut soin de ce malade $^{21}$. Je ne parle pas ici du grand nombre de lettres dont la maladie de Haller était le sujet.

Haller qui fut directeur des salines de Roche de 1758 à 1764 songeait souvent après son retour à Berne à accepter un poste à l'étranger qui lui rapporterait plus de revenus ${ }^{22}$. C'est ce que Tissot chercha à empêcher en soulignant auprès des Bernois les mérites de Haller pour sa patrie. Lorsqu'en janvier 1765 Carl Emanuel de Bonstetten (1706-1773) pria Tissot d'écrire un mémoire sur une réforme médicale, le plus important désir de Tissot fut la nomination de Haller comme directeur de l'hôpital de l'île ${ }^{23}$. Mais cette réforme ne fut jamais réalisée.

Haller à son tour empêcha de toutes ses forces le départ de Tissot lorsque celui-ci reçut en 1766 une offre du roi Stanislas-Auguste de Pologne qui l'appela 
auprès de lui comme médecin ordinaire. Le 26 janvier 1766 Haller écrivit à Tissot: «Il n'y a dans toute une province que Vous de medecin, et nous Vous perdrions. J'ai donné l'allarme toute chaude a un nombre de nos chefs; je tacherai de concerter avec M. le Tresorier de Bonstetten la maniere de Vous donner un signe de vie de la part de la Republique, qui puisse Vous conserver a la patrie; tout y est disposé. Je ferai agir d'un autre coté le Senat de Santé, et surement Vous aurés quelque marque distinctive de l'aprobation generale. ${ }^{24}$ Haller réussit à retenir Tissot à Lausanne en lui montrant les désavantages d'un séjour en Pologne et en déterminant le sénat de Berne à offrir à Tissot une chaire de professeur à l'Académie de Lausanne ${ }^{25}$. Tissot accepta et resta dans sa patrie en remerciant l'avoyer Albert-Frédéric d'Erlach (1696-1788) ${ }^{26}$. De plus Tissot reçut une rémunération. Haller écrivit le 25 juin 1767: «Vous allés recevoir 100 Ecus de Leurs Excellences, on a fait ecrire M. le Baillif, et la chose a passé gracieusement en Senat hier. On aura soin toutes les années, que la chose aille de meme. C'est peu de chose, mais le Senat ne peut faire d'avantage. ${ }^{27}$

Il n'y eut qu'une seule occasion pour Tissot d'être gravement en désaccord avec le gouvernement de Berne. Ce fut en 1769, lorsque les Bernois voulurent nommer le jurisconsulte Marc-Antoine Porta (1725-1797) professeur à l'Académie de Lausanne ${ }^{28}$. Porta avait été marié en premières noces avec Madame Tissot qui s'était après divorcée de lui pour son caractère indigne ${ }^{29}$. Tissot et ses amis lausannois firent jouer tous les ressorts pour empêcher la nomination de Porta. Ils virent une profonde humiliation de l'Académie dans ce témoignage d'honneur à un homme dont la conduite fut jugée licencieuse. Une lettre respectueuse fut adressée par l'Académie au gouvernement de Berne par l'entremise du bailli Vincent Tscharner (1722-1793) ${ }^{30}$. Dans leur réponse, l'avoyer Albert-Frédéric d'Erlach et le conseil de la ville de Berne exprimèrent leur extrême mécontentement et firent savoir au Lausannois que la lettre qu'ils avaient écrite n'était pas même digne de réponse. La lettre de Berne acheva d'indigner le public de Lausanne $^{31}$ et Tissot se plaignit auprès de Haller de cette affaire dans sa lettre du 29 avril 1769. Il exprimait l'opinion que l'Académie devrait au moins avoir le droit de faire des représentations ${ }^{32}$. Haller chercha à calmer son ami dans sa lettre du 3 mai 1769 en écrivant: «Ne pouriés Vous pas regarder le tout comme un triomphe passager d'une erreur, d'une demarche precipitée d'un Souverain d'ailleur trez bon et trez juste, mais qui n'a senti assez delicatement pour Vous. » ${ }^{33}$ Porta prit donc sa place dans l'Académie et Haller écrivit: «On doit pardonner bien des choses a sa patrie. ${ }^{34}$ Cependant Tissot ne montra plus beaucoup de zèle dans les affaires de l'Académie pour éviter une rencontre avec Porta $^{35}$. 
Mais ce désaccord fut passager et les bonnes relations de Tissot avec le patriciat bernois restèrent intactes. Après la mort de Haller, Tissot accepta la nomination de professeur à Pavie par l'empereur Joseph II. Il y enseigna pendant deux ans de 1781 à 1783 pour rendre possible l'étude de la médecine à son neveu qu'il avait adopté ${ }^{36}$. Après le retour de Tissot à Lausanne, le collège de médecine fut créé dans cette ville le 27 septembre 1787 par le gouvernement bernois ${ }^{37}$. Le bailli de Lausanne en fut le président et Tissot devint vice-président. Le collège devait en principe s'étendre sur l'inspection générale de tous ceux qui pratiquaient la médecine dans le Pays de Vaud, sur le contrôle des maladies contagieuses et la surveillance des vendeurs de drogues. En outre le collège devait s'attacher à découvrir tous les abus qui se seraient glissés dans l'exercice de la médecine et proposer au Conseil de santé les moyens d'y remédier. Tissot déploya une vive activité dans ce collège en écrivant bien des mémoires à la demande du gouvernement de Berne.

Vers la fin de la vie de Tissot, les idées de la révolution française commencèrent à se répandre. Le Vaudois Frédéric-César de la Harpe (1754-1838) agit à l'étranger en faveur de la libération de son pays. Mais Tissot ne partagea point ses opinions puisqu'il avait bien su profiter du gouvernement bernois dont il n'était pas mécontent ${ }^{38}$. Il ne demandait pas plus que le souverain ne pouvait donner et il trouvait que le patriciat de Berne était, à tout prendre, bienfaisant et paternel. Il avait des amis influents parmi les Bernois et il détesta la terreur dans la capitale française pendant la révolution qui fit tant de victimes. Après la publication de l'«Essai sur la constitution du Pays de Vaud» par La Harpe en 1797, Tissot rédigea des notes sous forme de lettre au colonel De La Harpe en 1797. Cette lettre se trouvait parmi les manuscrits de Tissot qui n'étaient pas destinés à la publication. Bien que Tissot feignît un instant de prendre La Harpe au sérieux, l'ironie n'est pas à méconnaître. Peu de temps après la rédaction de cette lettre, Tissot tomba malade et mourut le 13 juin 1797, fidèle ami et serviteur du gouvernement de Berne ${ }^{39}$. Représentant de l'ancien régime, Tissot n'avait jamais cherché qu'à servir le bien public et à être utile à ses compatriotes et surtout à ses malades.

\section{Annotations}

${ }^{1}$ Olivier, Eugène, Médecine et santé dans le pays de Vaud au XVIII ${ }^{e}$ siècle, Lausanne 1939,

Tome II, p.1060; Tome I, p.135.

${ }^{2}$ Eynard, Ch., Essai sur la vie de Tissot, Lausanne 1839, p. 24.

${ }^{3}$ l.c. p. $20-21$. 
${ }^{4}$ Hintzsche, Erich (éd.), Albrecht von Hallers Briefe an Auguste Tissot 1754-1777, Bern/ Stuttgart/Wien 1977, p. 25.

5 l.c. p. 26.

${ }^{6}$ l.c. p. 26-29.

${ }^{7}$ l.c. p.61, 70, 72, 78, 82, 129.

${ }^{8}$ l.c. p. $167,171$.

${ }^{9}$ Eynard, Ch., p. 35.

${ }^{10}$ Hintsche, E., p. 69, 71, 76.

11 l.c. p. 208, 210, 213.

12 l.c. p. 217.

${ }^{13}$ Tissot, Auguste, Avis au peuple sur sa santé, $2^{\text {me }}$ édition, Lausanne 1763, Préface.

${ }^{14}$ Eynard, Ch., p. 82-83.

${ }^{15}$ Olivier, Eugène, Tome I, p.1.

16 Eynard, Ch., p.111-112.

17 Hintzsche, E., p.191-195, 250, 302, 304.

${ }^{18}$ l.c. p. 320.

${ }^{19}$ l.c. p. $157-163$.

${ }^{20}$ l.c. p. 177.

${ }^{21}$ l.c. p. 332.

${ }^{22}$ l.c. p. $159,196,297-299,322$.

${ }^{23}$ l.c. p. 201-203.

${ }^{24}$ l.c. p. 223.

25 l.c. p. 225.

${ }^{26}$ l.c. p. 229.

27 l.c. p. 263.

28 l.c. p. 305.

${ }^{29}$ Eynard, Ch., p. 34 .

30 l.c. p. 204.

${ }^{31}$ l.c. p. 208-209.

${ }^{32}$ Hintzsche, E., p. 307.

33 l.c. p. 308.

${ }^{34}$ l.c. p. 310.

35 Eynard, Ch., p. 214.

${ }^{36}$ l.c. p. 286-315.

${ }^{37}$ Olivier, Eugène, Tome I, p. 18.

${ }^{38}$ Eynard, Ch., p.379-384.

${ }^{39}$ l.c. p. 387.

\section{Bibliographie (ordre chronologique)}

Ch. Eynard, Essai sur la vie de Tissot, Lausanne 1839.

Le deuxième centenaire du docteur Tissot (1728-1797). Revue historique Vaudoise 36 (1928), p. 226-320 (Contributions de A.Guisan, E.Olivier, M. et $M^{\mathrm{me}}$ de Sévery, G.-A. Bridel). 
Eugène Olivier, Médecine et santé dans le pays de Vaud au XVIII siècle, 1675-1798, Lausanne 1939.

Johannes Karcher, Über einige berühmte schweizerische Praktiker aus der Zeit der Aufklärung. Schweiz. med. Wschr.22 (1941), p. 887-889.

Heinrich Walther Bucher, Tissot und sein Traité des nerfs (Zürcher medizingeschichtliche Abhandlungen, Neue Reihe 1), Zürich 1958.

Geneviève Minder-Chappuis, Auguste Tissot. Sa correspondance avec A. de Haller et ses œuvres durant la période de 1754 à 1761. Thèse méd. Bern 1973.

Erwin H.Ackerknecht et Heinrich Buess, Kurze Geschichte der großen Schweizer Ärzte, Bern/Stuttgart/Wien 1975, p.39-42.

Erich Hintzsche (éd.), Albrecht von Hallers Briefe an Auguste Tissot, Bern/Stuttgart/Wien 1977.

\section{Summary}

Auguste Tissot (1728-1797) was born in the canton of Vaud which was subject to Bern since 1536. His family belonged to a class of wealthy people who were well connected with the patricians of Bern. Tissot who had studied medicine in Montpellier carried on a large correspondence with Albrecht von Haller (1708-1777) since the latter had returned from Göttingen to his native town Bern in 1753. The good services which these friends rendered each other were founded on mutual interest. On the initiative of Haller Tissot became professor at the Academy of Lausanne after having rejected in 1766 his appointment to the post of physician in ordinary to Stanislav Augustus, king of Poland. Tissot became famous throughout Europe when he wrote his "Avis au peuple sur sa santé». This book dealt with hygienics and medical care for the people living in the country. Especially the patricians of Bern made use of the services of Tissot. From 1781 to 1783 he was professor in Pavia when he was called there by the emperor Joseph II. After his return to Lausanne Tissot still remained a faithful servant of the sovereign of Bern even when the Vaudois patriot Frédéric-César de la Harpe (1754-1838) strove for the liberation of his country from the capital. Tissot did not live to see the creation of the canton of Vaud, for he died on June 13, 1797.

Dr. Marie-Louise Portmann

Medizinhistorische Bibliothek

Klingelbergstraße 23

CH-4031 Basel 